\title{
Ernst Kleinert: Mathematische Modelle des Kontinuums
}

\author{
Springer Spektrum 2019, VII + 165 Seiten, \\ ISBN: 978-3-662-59678-4, €24,99; eBook ISBN 978-3-662-59679-1, \\ $€ 19,99$
}

\section{Edmund Weitz}

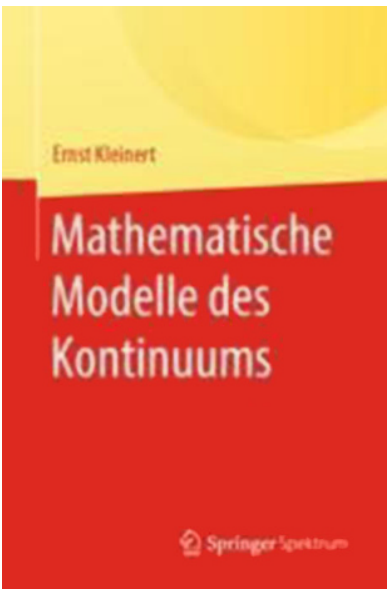

Gottfried Wilhelm Leibniz hätte sich über dieses Buch sicher gefreut. Hat er doch geschrieben, dass man ohne die Philosophie niemals auf den Grund der Mathematik dringt (und übrigens auch umgekehrt). Ernst Kleinert arbeitet in Mathematische Modelle des Kontinuums mit einer gelungenen Mischung aus Mathematik und Philosophie daran, zu diesem Grund vorzudringen. Leitfaden des Buches ist das namensgebende Kontinuum, über das die Philosophen seit der griechischen Antike nachdenken. Die Mathematik hat im Laufe der Zeit ganz unterschiedliche Theorien entwickelt, um das Kontinuum zu beschreiben und $\mathrm{zu}$ formalisieren, und acht davon werden von Kleinert vorgestellt und verglichen.

Den Anfang macht die von Eudoxos begründete und von Euklid verewigte Lehre von den Proportionen, die bekanntlich nötig wurde durch die Entdeckung inkommensurabler Verhältnisse. Proportionen sind die Verhältnisse „kompatibler“ Größen, beispielsweise von Längen zu Längen. Für uns sind das heute einfach reelle Zahlen, aber mehr als 2000 Jahre lang war die Bezeichnung Zahl exklusiv für die Objekte reserviert, die wir inzwischen natürliche Zahlen nennen.

Alle weiteren Systeme sind wesentlich neueren Datums. Zumindest drei dürften vielen Lesern bekannt vorkommen. Von Hilbert stammt die Idee der Gewinnung algebraischer Strukturen aus axiomatisierter Geometrie: Auf Mengen von Punk-

E. Weitz (殴

Hamburg University of Applied Sciences, Hamburg, Deutschland

E-Mail: edmund.weitz@haw-hamburg.de 
ten werden durch geometrische Konstruktionen Rechenoperationen definiert, die schließlich zu einem (also dem) vollständigen angeordneten Körper führen. Das heutige „Standardmodell“" nach Dedekind, Cantor, Méray und Heine wird oft bereits in Erstsemestervorlesungen unter dem Namen Aufbau des Zahlensystems vorgeführt: Im letzten Schritt werden die reellen Zahlen als Schnitte in der Menge der rationalen Zahlen oder als (Äquivalenzklassen von) Fundamentalfolgen eingeführt. In der Nichtstandardanalysis, die einen etwas unglücklichen Namen trägt, wird die Menge der reellen Zahlen mit Methoden aus Logik und Mengenlehre um ,unendlich kleine“ und „unendlich große“ Zahlen erweitert, wodurch im Nachhinein die infinitesimalen Größen von Leibniz und die heutzutage teilweise haarsträubend anmutenden Berechnungen Eulers rehabilitiert werden.

Aus dem aktuellen Jahrtausend stammt der Ansatz von A'Campo, der ganz ohne rationale Zahlen auskommt. Die reellen Zahlen werden hier über Funktionen definiert, die ganze Zahlen auf ganze Zahlen abbilden und die sich fast wie lineare Funktionen verhalten. Und beinahe exotisch wirken auf die Nichteingeweihten die sogenannte synthetische oder glatte Infinitesimalrechnung sowie Conways System der surrealen Zahlen. Conways Zahlen, die ursprünglich zur Analyse von Spielen entwickelt wurden, werden durch einige wenige Regeln rekursiv im gewissen Sinne ,aus dem Nichts“ gezaubert und man erhält als Ergebnis eine echte Klasse von Objekten, die unter anderem die reellen Zahlen, infinitesimale Zahlen und transfinite Ordinalzahlen umfasst. Die synthetische Infinitesimalrechnung verwendet Methoden aus der Kategorientheorie. Wie in der Nichtstandardanalysis gibt es infinitesimale Größen, aber diese haben keine Kehrwerte. Außerdem arbeitet man nicht mit der klassischen Logik, sondern verzichtet beispielsweise auf das Tertium non datur. Philosophisch ist dieser Ansatz insbesondere deswegen interessant, weil er im Gegensatz zu den anderen modernen nicht atomistisch ist: Das Kontinuum besteht nicht aus diskreten Punkten, sondern aus infinitesimalen Streckenstücken.

Schließlich bildet als „Kontrapunkt“ Brouwers intuitionistische Theorie der reellen Zahlen den Abschluss und erinnert an eine Zeit, in der die Natur des Kontinuums noch heftig diskutiert wurde. Auch hier wird die Logik eingeschränkt. (Für Hilbert war das, als würde man ,,dem Boxer den Gebrauch der Fäuste untersagen“.) Außerdem spricht man nur über Objekte, für die man eine explizite Konstruktionsregel angeben kann.

Das alles kann man sicher auch einzeln und ausführlicher an anderen Stellen finden - wenn man weiß, wonach man suchen muss. In dieser konzentrierten Zusammenstellung und eingebettet in die scharfsinnigen und sachkundigen Kommentare des Verfassers ist es aber ein Novum. Abgeschlossen wird jedes Kapitel durch Empfehlungen für vertiefende Lektüre, bei denen es Kleinert offenbar mehr um Qualität als um Quantität ging.

Man kann sich zusammenreimen, dass so viel Stoff auf den 140 Seiten, die zwischen Einleitung und Zusammenfassung verbleiben, nicht in epischer Breite ausgearbeitet werden kann. Definitionen, nummerierte Lemmata und Theoreme, deren Beweis mit q.e.d. endet, sollte man nicht erwarten. Alle Themen werden jedoch so weit behandelt, dass es für einen soliden Eindruck reicht. Mitbringen sollte man eine gewisse „mathematische Reife“, die man typischerweise nach den ersten drei, vier Semestern eines Mathematikstudiums erworben hat, sowie die Bereitschaft, 
mit Zettel und Bleistift mitzuarbeiten und Gedankengänge selbstständig zu vollenden. Zudem wird man wohl ab und zu etwas nachschlagen müssen. Für Menschen, die mit der formalen Denkweise der Grundlagenmathematik fremdeln, könnte die zweite Hälfte des Buches eine Herausforderung sein. (Und gerade das längste und abstrakteste Kapitel - das über die synthetische Infinitesimalrechnung - scheint mir didaktisch das am wenigsten gelungenste.)

Kleinert schreibt im Vorwort, dass er im Buch ,den Vorlesungston mit seiner direkten Ansprache beibehalten" habe. Und in der Tat kommt dann ein paar Seiten später unvermittelt so etwas wie „dieses Stück Kreide“ vor und man muss sich wohl vorstellen, dass der Autor in einem Vortrag, der dem Kapitel zugrunde lag, ein Stück Kreide in die Höhe hielt. Größtenteils wirkt der Text jedoch so, als wäre jedes Wort auf der Goldwaage geprüft und im Zweifelsfall gestrichen oder durch ein passenderes ersetzt worden. Der Duktus ist dichter, variantenreicher und komplexer als der, den man aus typischen Mathebüchern kennt. Wenn das wirklich der O-Ton der Vorlesung war, dann wurde wahrscheinlich vom Manuskript abgelesen und das Auditorium war zu höchster Konzentration verurteilt, da man einen gesprochenen Satz schwerlich ein zweites Mal lesen kann.

Es liegt nahe, das hier besprochene Werk mit Oliver Deisers Reelle Zahlen zu vergleichen, das 2008 in den Semesterberichten zu recht gelobt wurde und das man inzwischen kostenlos von Deisers Website aleph1. info herunterladen kann. Beide Bücher haben das Kontinuum zum Thema und beide sprechen Leser an, die über den Tellerrand des Tagesgeschäftes schauen wollen. Erfreulicherweise konkurrieren sie jedoch nicht, sondern ergänzen sich. Abgesehen von den inzwischen klassischen Konstruktionen der reellen Zahlen und der von A'Campo gibt es kaum inhaltliche Überschneidungen und auch an diesen Stellen sind die Herangehensweisen sehr unterschiedlich. Deisers Buch ähnelt vom Stil her mehr einem Lehrbuch, während Kleinert wie oben schon angedeutet fordernder ist und oft eher skizziert als ausarbeitet. Und obwohl auch Deiser auf die historische Entwicklung eingeht, ist bei ihm das Ziel der Erörterungen doch die mathematische Durchdringung der Themen, während die Mathematik bei Kleinert Ausgangspunkt philosophischer Betrachtungen ist. (Allerdings ist Deisers Buch dadurch auch ungefähr dreimal so lang.)

Auf jeden Fall sollte es mehr Bücher wie diese geben! Doch wer soll sie lesen? Idealiter wohl die, die Mathematik studieren. Es ist jedoch zu befürchten, dass solche Texte durch das Raster der Bologna-Reform fallen, weil in der eng getakteten Welt der Module und Credit Points für sie kein Platz ist. Und für das Studium generale und die meisten interessierten Laien dürften sie mathematisch zu anspruchsvoll sein. Aber vielleicht (hoffentlich!) ist diese Sichtweise ja auch zu pessimistisch.

Wenn es an Kleinerts Werk etwas auszusetzen gibt, dann dies: Wäre Springer noch der Verlag, der er im letzten Jahrhundert mal gewesen ist, dann hätte den Autor eine typographisch versierte Person unterstützt, die das Buch etwas liebevoller und lesefreundlicher gesetzt hätte. Und ein Lektor hätte das Manuskript tatsächlich gelesen und vor der Veröffentlichung die eine oder andere kleine Schludrigkeit bemängelt. (Man kann es aber auch positiv wenden und solche Petitessen lernpsychologisch als desirable difficulties einordnen.)

Buchbesprechungen erwecken manchmal den Eindruck, der Rezensent wisse mehr als der Verfasser des Buches oder maße sich an, ein besseres Buch schrei- 
ben zu können. Das ist hier definitiv nicht der Fall. Der Autor dieser Zeilen hat bei der Lektüre des Buches Neues gelernt und wurde zum Nachdenken angeregt. Er hat es mit viel Vergnügen gelesen und kann es wärmstens jedem empfehlen, der seinen Horizont erweitern und der Mathematik im Leibnizschen Sinne ,auf den Grund dringen“" will. Und insbesondere jeder Studierende der Mathematik sollte das wollen!

Funding Open Access funding enabled and organized by Projekt DEAL.

Open Access Dieser Artikel wird unter der Creative Commons Namensnennung 4.0 International Lizenz veröffentlicht, welche die Nutzung, Vervielfältigung, Bearbeitung, Verbreitung und Wiedergabe in jeglichem Medium und Format erlaubt, sofern Sie den/die ursprünglichen Autor(en) und die Quelle ordnungsgemäß nennen, einen Link zur Creative Commons Lizenz beifügen und angeben, ob Änderungen vorgenommen wurden.

Die in diesem Artikel enthaltenen Bilder und sonstiges Drittmaterial unterliegen ebenfalls der genannten Creative Commons Lizenz, sofern sich aus der Abbildungslegende nichts anderes ergibt. Sofern das betreffende Material nicht unter der genannten Creative Commons Lizenz steht und die betreffende Handlung nicht nach gesetzlichen Vorschriften erlaubt ist, ist für die oben aufgeführten Weiterverwendungen des Materials die Einwilligung des jeweiligen Rechteinhabers einzuholen.

Weitere Details zur Lizenz entnehmen Sie bitte der Lizenzinformation auf http://creativecommons.org/ licenses/by/4.0/deed.de. 